\title{
Double-electron ionization driven by inhomogeneous fields
}

A. Chacón ${ }^{1 \star}$, L. Ortmann ${ }^{2}$, F. Cucchietti ${ }^{3}$, N. Suárez ${ }^{1}$, J. A. Pérez-Hernández $^{4}$, M. F. Ciappina ${ }^{5}$, A. S. Landsman ${ }^{2,6}$, M. Lewenstein ${ }^{1,7}$

1 ICFO-Institut de Ciències Fotòniques, The Barcelona Institute of Science and Technology, Av. Carl Friedrich Gauss 3, 08860 Castelldefels (Barcelona), Spain

2 Max Planck Institute for the Physics of Complex Systems, Nöthnitzer Straße 38, D-01187 Dresden, Germany

3 Barcelona Supercomputing Center BSC-CNS, Campus Nord UPC, Barcelona, Spain

4 Centro de Láseres Pulsados (CLPU), Parque Científico, E-37185 Villamayor, Salamanca, Spain

5 Institute of Physics of the ASCR, ELI-Beamlines, Na Slovance 2, 18221 Prague, Czech Republic

6 Max Planck Postech/Department of Physics, Pohang, Gyeongbuk 37673, Republic of Korea

7 ICREA, Pg. Lluís Companys 23, 08010 Barcelona, Spain

Received: date / Revised version: date

\begin{abstract}
Electron-electron correlation effects play an crucial role in our understanding of sequential (SDI) and non-sequential double ionization (NSDI) mechanisms. Here, we present a theoretical study of NSDI driven by plasmonic-enhanced spatial inhomogeneous fields. By numerically solving the time-dependent Schrödinger equation for a linear reduced model of He and a double-electron time-evolution probability analysis, we provide evidence for the enhancement effects in NSDI showing that the double ionization yield at lower laser peak intensities is increased due to the spatial inhomogeneous character of plasmonic-enhanced field. The change in the emission direction of the double-ion as a function of the field inhomogeneity degree demonstrates that plasmonic-enhanced fields could configure a reliable instrument to control the ion emission. Furthermore, our quantum mechanical model, as well as classical trajectory Monte Carlo simulations, show that inhomogeneous fields are as well as a useful tool for splitting the binary and recoil processes in the rescattering scenario.
\end{abstract}

* alexis.chacon@icfo.eu 


\section{Introduction}

Since 1982, when L'Huillier presented the first experimental observation of a large enhancement in the double-charge ionization yield of Xe driven by an intense infrared (IR) laser-field, a number of questions about electronelectron (e-e) correlation effects and their mechanisms have arisen $[1,2]$. The fact that those results could not be explained in the framework of SDI, where e-e correlation effects are assumed negligible, opened the path of considering the importance of such correlation effects in the ionization processes [1-5]. It was then that the concept of Non-Sequential Double Ionization (NSDI) arose as an explanation of the 1982 experiment [6-9]. However, in the NSDI mechanism there are several processes such as the shake-off, laser-field-assisted rescattering ionization, Rescattering Impact Double Ionization (RIDI) [1013] and Rescattering Excitation with Subsequent Ionization (RESI), which might take place during the DI of atoms. The question of how to disentangle RIDI and RESI (and within RIDI the binary and recoil processes) are therefore still under investigation in the attosecond science community [3].

The most important mechanisms behind the NSDI process driven by a spatially homogeneous strong laser field in the midinfrared regime are the RIDI and the RESI [9]. The importance of each of them basically depend on: (1) the gas atomic (or molecular) target and (2) the field features. The RIDI mechanism occurs within the so called rescattering scenario. According to Corkum [10], once the first electron is launched into the continuum, this process happens about the maximum of the driven laser field via tunneling, this electron accumulates a kinetic energy $E_{1 k}$ and has certain probability to come back to the vicinity of ion core. At this rescattering time, and if the electron kinetic energy is larger than the second ionization potential $\left(I_{2 p}\right)$ of the remaining electron $\left(E_{1 k} \geq I_{2 p}\right)$, the second electron is kicked out from the target "instantaneously" (see Fig. 1(a)).

However, in case that the collided first electron does not have enough energy to knock out the second electron, (i.e. $E_{1 k}<I_{2 p}$ ), this remaining electron should have a certain probability to be excited from its ground state to another excited state and will not be instantaneously ionized. Nevertheless, at a subsequent maximum of the oscillating laser field, this second excited electron can be indeed ionized via tunneling (see Fig. 1(b)). The latter process is known as RESI.

Prior studies addressing e-e correlation effects in laser-driven multiple ionization processes were done considering only spatially homogeneous fields, i.e. fields that do not present spatial variations in the region where the electron dynamics takes place. This is a legitimate assumption considering that in conventional laser-matter experiments the laser electric field changes in a region on the orders of micrometers, whereas the electron dynamics develops on a nanometric scale. However, since recent studies of 

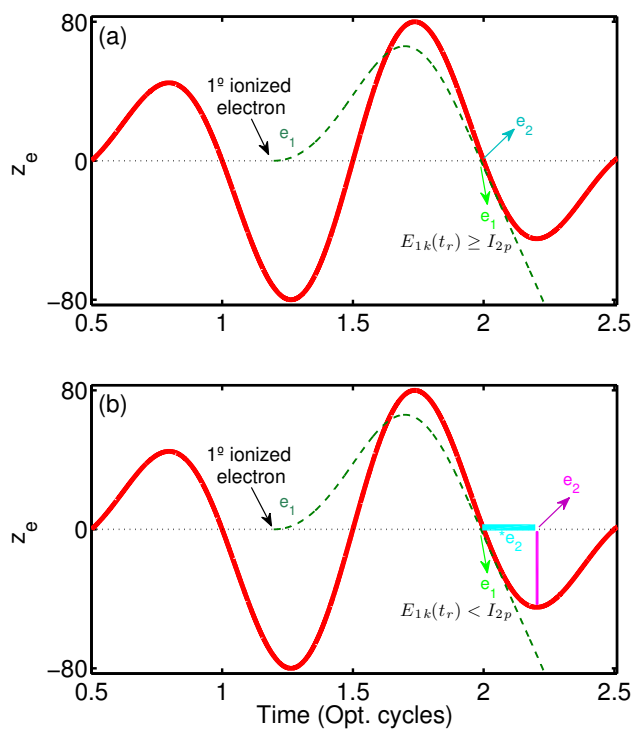

Fig. 1 Panels (a) and (b) show the classical pictures of the rescattering impact double ionization (RIDI) and rescattering excitation with subsequent double-ionization (RESI) scenarios, respectively. The red continuum and green dashed lines denote the IR laser field oscillations and the trajectory of the first ionized electron about $t \approx 1.25 T_{0}\left(T_{0}\right.$ is the period of the laser optical field), respectively. In (a) the light green and light blue arrows indicate the first and second ionized electrons. Note that the second electron is launched into the continuum about $t \approx 2 T_{0}$ when $E_{1 k} \geq I_{2 p}$. In (b), at the recollision time, denoted by a light green arrow $\left(t \approx 2 T_{0}\right)$, the second electron is excited and remains in this state, noted by a light blue line, until it is laser-ionized by tunneling at a subsequent maximum of the laser field (denoted by a magenta arrow) about $t \approx 2.25 T_{0}$.

post-ionization dynamics in spatially inhomogeneous fields [14] provides new physical effects and insights, a question arises as to the influence of spatial variation on the DI process. The aim of this work is to present a complete study of DI driven by plasmonic-enhanced spatially inhomogeneous fields with an investigation of NSDI in general, and the RESI and RIDI mechanisms in particular. Plasmonic-enhanced spatially inhomogeneous fields appear when a short laser pulse, of low intensity, illuminates a metallic or dielectric nanostructure. As a consequence of the formation of surface plasmon polaritons (SPPs), an amplification (enhancement) of the incoming laser electric field takes place. This enhancement is high enough to allow the development of strong laser-matter phenomena, as high-order harmonic generation (HHG), above-threshold ionization (ATI) and DI, amongst others. In addition, due the nanometric dimensions of the so-called hot spots, both the laser electric field and the associated vector potential present spa- 
tial variations in a scale comparable to the one where the electron develops its motion. As a consequence, the theoretical approaches should to incorporate this new feature in the laser-matter coupling (for a comprehensive review of recent theoretical and experimental developments see e.g. [15]).

We will employ both quantum mechanical approaches, based on the numerical solution of the time-dependent of the Schrödinger equation (TDSE) for two electrons in reduced dimensions, and classical schemes employing classical trajectories Monte Carlo (CTMC) simulations to deal with DI driven by plasmonic-enhanced spatially inhomogeneous fields. Within the quantum framework, we employ a linear model for the helium atom, where the motion of both electrons is restricted to the direction of the laser polarization. Experience has shown that 1D models qualitatively reproduce strong-field phenomena such as the double-ionization knee structure $[16,17]$ or above-threshold ionization [18] and intense-field double ionization mechanisms [19].

The rest of this paper ${ }^{1}$ is organized as follows. In the next section we present our theoretical tools, namely the TDSE and CTMC for two electrons in reduced dimensions. Then, in Section 3, we show a comparative study between DI driven by conventional laser pulses and DI governed by plasmonic-enhanced spatially inhomogeneous fields. We put particular emphasis on the two-electron momentum distribution, considering it represents one of the most detailed observables and is fully experimentally accessible. We end up with concluding remarks and a brief outlook.

\section{Numerical model}

We study the two-electron dynamics driven by plasmonic-enhanced fields via a fully quantum mechanical linear model of the helium atom and the integration of Newton's equations in the framework of the CTMC method. The $a b$-initio quantum mechanical calculations allow us to address the whole electron-electron (e-e) correlated dynamics by means of the numerical solution of the TDSE similarly to those used by Lein [19] and Watson [23,18]. The Hamiltonian of our 2e system reads (atomic units are used throughout

1 This contribution is dedicated to Ted Hänsch on the occasion of his 75 th birthday. Although Prof. Hänsch is mostly regarded for "contributions to the development of laser-based precision spectroscopy, including the optical frequency comb technique", and his contributions to laser cooling and physics of ultracold atoms, his influence on attosecond physics is hard to underestimate. For instance, he predicted at very early stages the possibility of generating attosecond pulse trains from phase locked harmonics $[20,21]$ and pioneered and contributed to the initial studies of the high-order harmonics coherence [21,22]. His group has also developed decisive steps extending the frequency combs toward high frequencies regimes. 
the paper unless specified otherwise)

$$
H=\sum_{j=1}^{2}\left[\frac{p_{j}^{2}}{2}+V\left(z_{j}\right)+V_{\mathrm{int}}\left(z_{j}, t\right)\right]+V\left(z_{1}, z_{2}\right),
$$

where $p_{j}=-i \frac{\partial}{\partial z_{j}}$ is the momentum operator corresponding to the $j$-th electron ( $j$-th-e), $j=1,2 . V\left(z_{j}\right)=-\frac{Z}{\sqrt{z_{j}^{2}+a}}$ and $V\left(z_{1}, z_{2}\right)=\frac{1}{\sqrt{\left(z_{1}-z_{2}\right)^{2}+b}}$ are the attractive potential of the interaction of the $j$-th electron with the nucleus of charge $Z$ and the repulsive e-e potential, respectively. The potential describing the interaction of the $j$-th electron with the spatially dependent laser electric field in length gauge is [24]

$$
V_{\mathrm{int}}\left(z_{j}, t\right)=\left(z_{j}+\frac{\beta}{2} z_{j}^{2}\right) E_{\mathrm{h}}(t),
$$

where $\beta$ denotes the inhomogeneity strength (see e.g. $[24,25]$ for more details) of the plasmonic field and $E_{\mathrm{h}}(t)=E_{0} \sin ^{2}\left(\omega_{0} t / 2 \mathrm{~N}\right) \sin \left(\omega_{0} t+\varphi_{0}\right)$ is the spatially homogenous or conventional laser electric field. Here, $E_{0}, \omega_{0}, \mathrm{~N}$ and $\varphi_{0}$ are the laser electric peak amplitude, laser frequency, total number of cycles and carrier envelope phase (CEP), respectively.

The numerical algorithm used to solve the TDSE for our linear 1Dx1D He model is the Split Operator method described in Ref. [26,27]. This algorithm takes advantage of the Fast Fourier Transform (FFT) paradigm to evaluate the kinetic energy operators of Eq. (1) in the Fourier space. To speed up our calculations and redistribute the whole $2 \mathrm{e}$ wavefunction in position space-with a total number of points $N_{T}=N_{1} \times N_{2} \approx 4 \times 10^{4} \times 4 \times 10^{4}=$ $1.6 \times 10^{9}$ - on different computational nodes, $N_{p}$, we employ the message passing interface MPI parallelized version of the FFTW [28]. This implementation allows us to reach large electron excursions $z_{j} \gg \frac{E_{0}}{\omega_{0}^{2}}$, which is typical for electrons driven by spatially inhomogeneous fields [24,25]. Each 1Dx1D TDSE calculation took about 11735 CPU-hours on $N_{p}=1024$ cores in the Barcelona Supercomputer Center.

For the He linear model, we have fixed the soft-core parameters and the nucleus-charge to $a=b=1$ a.u., and $Z=2$, respectively. With these values, we obtain a $2 \mathrm{e}$ ground state energy of $E_{1,2}=-2.238$ a.u. Although the matching with the experimental data is not perfect, it is sufficient to qualitatively reproduce the 2e-dynamics driven by a linearly polarized laserfield $[19,23,18,29]$. The $2 \mathrm{e}$ ground state wavefunction $\Psi_{0}\left(z_{1}, z_{2}\right)$ is obtained via imaginary-time propagation - with an imaginary time step $\delta t=-i 0.025$ a.u. - switching off the interaction potential in Eq. (2), i.e. $V_{\text {int }}\left(z_{j}, t\right)=0$. The first electron ionization and the second electron ionization potentials are then $I_{1 p}=0.751$ a.u. and $I_{2 p}=1.487$ a.u., respectively.

In order to follow the 2e dynamics driven by the plasmonic-enhanced spatially inhomogeneous fields, encoded in the the time-dependent wavefunction $\Psi\left(z_{1}, z_{2}, t\right)$, the wavefunction $\Psi_{0}\left(z_{1}, z_{2}\right)$ of the ground state is prop- 


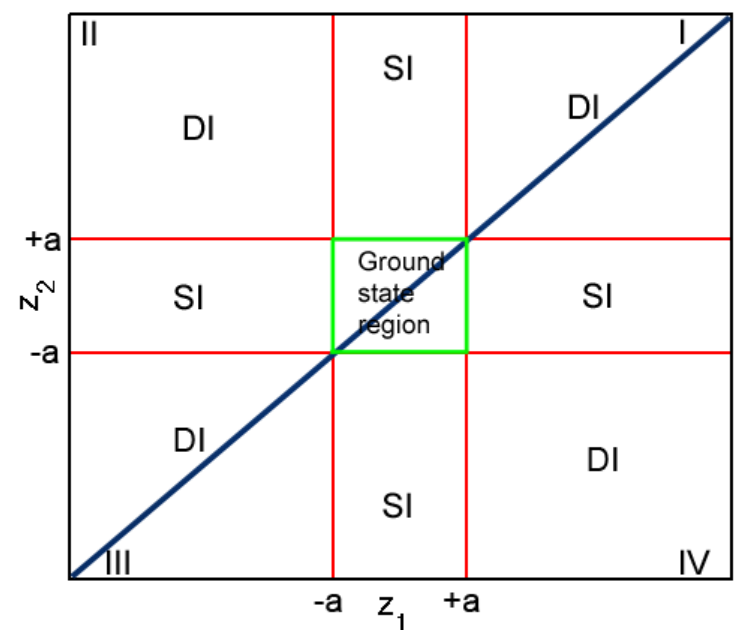

Fig. 2 Diagram of the different regions for the whole two-electron wavefunction in the position space $\left(z_{1}, z_{2}\right)$. The entire region can be split in $(i)$ the spatial ground state area, (ii) the single-electron ionization (SI) area and (iii) the double-electron ionization (DI) area (see the text and consider $z_{a}=a$ ). Note that the (i) spatial region is graphically described by the green central square, the $(i i)$ is the inner region within the red horizontal and vertical lines without considering $(i)$, and the (iii) region is the remaining area, including parts in the I, II, III and IV quadrants. The diagonal dark blue line points out the antisymmetric condition for the two-electron systems.

agated in real time via TDSE with the Hamiltonian defined in Eq. (1). In addition, we compute the single-electron ionization, $P_{1 \mathrm{e}}(t)$, and 2e-ionization, $P_{2 \mathrm{e}}(t)$, as a function of time $t$. The 2e position distribution $\left|\Psi\left(z_{1}, z_{2}, t\right)\right|^{2}$ is split into three parts: (i) $\left\{\left|z_{1}\right|,\left|z_{2}\right|<z_{a}\right\}$, (ii) $\left\{\left|z_{1}\right|<z_{a},\left|z_{2}\right| \geq z_{a}\right\}$ or $\left\{\left|z_{1}\right| \geq z_{a},\left|z_{2}\right|<z_{a}\right\}$, and (iii) $\left\{\left|z_{1}\right|,\left|z_{2}\right| \geq z_{a}\right\}$ with $z_{a}=90$ a.u..

As it is illustrated in Fig. 2, the first region $(i)$ describes the 2e bound wavefunction, $\Psi_{b}\left(z_{1}, z_{2}, t\right)$, part of $\Psi\left(z_{1}, z_{2}, t\right)$. The second one (ii) defines the single-electron ionization (SI) $\Psi_{1 \mathrm{e}}\left(z_{1}, z_{2}, t\right)$, which is the time dependent $\mathrm{He}^{+}$yield. And the third region (iii) includes the doubleelectron ionization (DI) $\Psi_{2 \mathrm{e}}\left(z_{1}, z_{2}, t\right)$ part, which represents the $\mathrm{He}^{2+}$ time dependent yield production. Then, by integrating over regions (ii) and (iii) the single- and double-electron ionization $P_{1 e}(t)$ and $P_{2 e}(t)$ rates $\left(\mathrm{He}^{+}\right.$and $\mathrm{He}^{2+}$ production yields) are computed, respectively.

The final two-electron momentum distribution $\mathrm{S}_{2 \mathrm{e}}\left(p_{1}, p_{2}\right)=\left|\Psi_{2 \mathrm{eM}}\left(p_{1}, p_{2}, t_{\mathrm{F}}\right)\right|^{2}$ is evaluated half a laser cycle after the end of the IR laser field as the absolute square of the projection of the final 2e wavepacket $\Psi_{2 \mathrm{e}}\left(z_{1}, z_{2}, t_{\mathrm{F}}\right)$ on the double-electron plane waves $\Phi_{p_{1}, p_{2}}\left(z_{1}, z_{2}\right)=\frac{1}{2 \pi} \exp \left[i\left(z_{1} p_{1}+z_{2} p_{2}\right)\right][19,11]$. Convergence tests on time propagation at $\frac{1}{3} T_{0}, \frac{1}{2} T_{0}$ and $T_{0}$ after the end of the oscillating laser fields shows that the $2 \mathrm{e}$ probability dis- 
tribution does not change considerably between $\frac{1}{2} T_{0}$ and $T_{0}\left(T_{0}\right.$ is the cycle period of the IR laser beam). In order to reduce the computational calculation time and position number of grid points, we chose as detection time half a cycle after the end of our oscillating field. Furthermore, the correlated ion $S_{\mathrm{He}^{2+}}\left(p_{\text {ion }}\right)$ momentum distribution is calculated by projecting $S_{2 \mathrm{e}}\left(p_{1}, p_{2}\right)$ on the diagonal $p_{1}=p_{2}$, which corresponds to the total 2e momentum $p=p_{1}+p_{2}$. Thereby, via momentum conservation of the system, the ion momentum reads $p_{\text {ion }}=-\left(p_{1}+p_{2}\right)$.

In order to supplement the quantum mechanical calculations and understand the physical origin of the effects of the plasmonic-enhanced field better, we implement CTMC simulations to investigate electron trajectories after ionization of helium under the so called RIDI mechanisms. The simulations are restricted to one dimension, namely the direction of field polarization, in which also the field inhomogeneity develops. The trajectories are launched at a starting time $t_{0}$, which is distributed probabilistically following the Ammosov-Delone-Krainov (ADK) formula [30,31], typically used to model strong field ionization [32-35]

$$
P\left(t_{0}, v_{\perp}\right)=\exp \left(-\frac{2\left(2 I_{p}\left(t_{0}\right)\right)^{3 / 2}}{3 E_{\mathrm{h}}\left(t_{0}\right)}\right)
$$

corresponding to an atom centered at the origin. $I_{p}$ denotes the Stark shifted ionization potential [36]

$$
I_{p}\left(t_{0}\right)=I_{1 p}+\frac{1}{2}\left(\alpha_{N}-\alpha_{I}\right) E_{\mathrm{h}}\left(t_{0}\right)^{2},
$$

with $\alpha_{N}$ and $\alpha_{I}$ representing the polarizability of the atom and ion, respectively. The tunnel exit radius is assumed to be zero following the simple man's model [10]. The dynamics of each electronic trajectory after ionization is solved numerically by integrating the Newton's equations of motion, which takes into account the laser field, but not the Coulomb potential following the model in [11].

If the electron returns to the ion $(z=0)$ at time $t_{r}$ with kinetic energy $E_{k 1}\left(t_{r}\right)$ larger than the ionization potential $I_{2 p}$ of the second electron [18], this second electron can be ionized as well. In this ionization process the kinetic energy of the first electron is reduced by $I_{2 p}$ and the second electron is born in the continuum with zero velocity

$$
\begin{aligned}
& p_{1}\left(t_{r}\right)= \pm \sqrt{2\left(E_{k 1}\left(t_{r}\right)-I_{2 p}\right)} \\
& p_{2}\left(t_{r}\right)=0 .
\end{aligned}
$$

Here, the two different signs in $p_{1}$ describe the possibility of scattering the first electron into forward, binary, or backward, recoil, direction with respect to its momentum directly before the ionization of the second remaining electron.

For each double ionization event, we calculated both options. The dynamics after the second ionization is again determined by the propagation 
in the laser field, where the Coulomb force is completely neglected [11]. The reason for doing so is the fact that close to the ion quantum effects play a crucial role, which cannot be captured in our classical model. Therefore, we restrict ourselves to the classical dynamics in the laser field. For the electron dynamics far away from the ion, this is not a problem anyway since here the Coulomb force is negligible, which is why it is common practice to treat the Coulomb force in this regime perturbatively [37].

\section{Double-electron ionization}

To study the e-e correlation effects we firstly compute the final single- and double-electron ionization yields as a function of the peak laser field intensity for a few-cycle IR pulse. This allows us to identify the intensity regions where the spatially inhomogeneous field substantially modifies the double-electron ionization process. Because of possible damage and ablation of the nanostuctures, we restrict ourselves to relatively low peak plasmonic-enhanced intensities: $I_{0}<10^{15} \mathrm{~W} / \mathrm{cm}^{2}$ (for more details about the parameters range and experimental constraints see e.g. [15]). Secondly, we compute the two-electron momentum distribution as a function of the inhomogeneity strength at a fixed laser intensity. This scan on the $\beta$ parameter provides enough evidence about the role of the spatial inhomogeneous field in the 2e ionization process. Furthermore, we scrutinise if the e-e correlation features are affected by the plasmonic-enhanced driven field.

\section{$3.1 \mathrm{He}^{+}$and $\mathrm{He}^{2+}$ ion yields}

We numerically compute the final 2e-ionization yield by the procedure described in Section 2. The grid parameters used in those calculations are $N_{1}=N_{2}=40960$ points and $\delta z_{1}=\delta z_{2}=0.25$ a.u. The integration time step was chosen $\delta t=0.025$ a.u. The results of the single- and double-electron ionization yield as a function of the peak laser field intensity for the homogeneous $(\beta=0)$ and inhomogeneous fields with $\beta=0.005$ a.u., are depicted in Fig. 3.

An enhancement of the final 2e ionization $P_{2 \mathrm{e}}\left(t_{\mathrm{F}}, I_{0}\right)$ is observed for the inhomogeneous field case when compared to the conventional one. Similar effects are also obtained in the comparison with the single-electron ionization yields. This feature is hardly visible in the Fig. 3, due to the logarithmic scale, although we have found an increment up to a factor of 4 in the double ionization yield. This enhancement clearly shows that the spatially inhomogeneous fields play an instrumental role in the NSDI of helium.

Naturally, the question about the origin of this enhancement arises. In order to answer it, we compute the single- $P_{1 \mathrm{e}}(t)$ and double-electron $P_{\mathrm{e} 2}(t)$ 


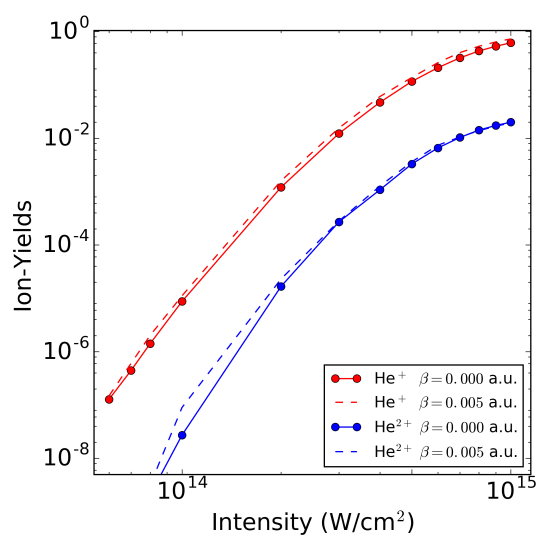

Fig. 3 Numerical TDSE calculations of the single- (red) and double-electron (blue) ionization yields of our linear 1Dx1D He 2e-model driven by homogeneous (line with circles) and inhomogeneous (dashed line) fields as a function of the laser peak intensity. The mean frequency of the IR laser field is $\omega_{0}=0.057$ a.u. $(1.55 \mathrm{eV})$, the CEP is $\varphi_{\mathrm{CEP}}=0^{\circ}$ and the total number of cycles is $\mathrm{N}=4$ under a $\sin ^{2}$ envelope.

ionization yield as a function of time at a fixed peak intensity of $I_{0}=2 \times$ $10^{14} \mathrm{~W} / \mathrm{cm}^{2}$. Here we focus our attention on the intensity region where the double ion yield, $\mathrm{He}^{2+}$, is enhanced by the inhomogeneous field. According to Fig. 3, one such region is $I_{0}=1-5 \times 10^{14} \mathrm{~W} / \mathrm{cm}^{2}$. The results of the time-evolved probabilities are depicted in Fig. 4.

For the single-electron ionization $P_{1 \mathrm{e}}(t)$ shown in Fig. 4(a), the "inhomogeneous" ionization yield is larger than the conventional one, in particular, at about 2.5 cycles of the IR laser. We could trace out the origin of this observation in a much stronger distortion of the laser-atomic potential barrier, which raises the probability of the first bound electron to 'escape' from the atom.

Fig. 4(b) shows a comparison of $P_{2 \mathrm{e}}(t)$ for conventional and inhomogeneous fields. About 3.4 cycles of the IR laser oscillations, the 2e ionization yield largely increases for the inhomogeneous field case with respect to the conventional one by more than 5 -times. At this very low inhomogeneity degree of $\beta=0.005$ a.u., and low IR peak intensity, this enhancement of the 2e-ionization rate is a very surprising result. Similar behaviour was previously observed in [14], where the double-electron ionization reaches higher yields leading to an enhancement in the intensity of the HHG signal. However, in that latter case a larger inhomogeneity degree of $\beta=0.02$ a.u was used.

An hypothesis that might explain that result is based on the three step Corkum's model $[6,9,10]$ where: firstly, the first electron ionizes via tunnelling, secondly, this electron propagates in the continuum gaining energy from the laser field - in our case a spatially inhomogeneous field - and then 

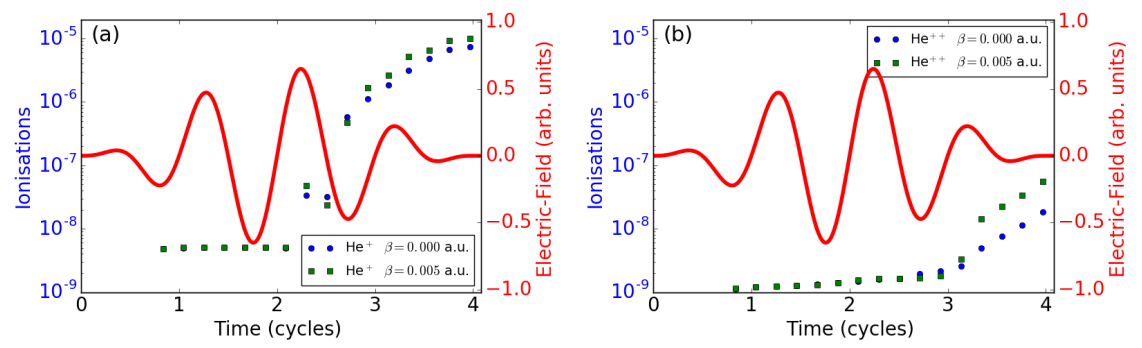

Fig. 4 Single- (a) and double-electron (b) ionization yields of our linear 1Dx1D He 2e-model driven by conventional and spatially inhomogeneous fields as a function time (see left axis). The IR laser field oscillations are depicted in red solid line. The laser peak intensity used to follow the two-electron dynamics is $I_{0}=2 \times 10^{14} \mathbf{W} / \mathbf{c m}^{2}$. The other laser parameters are the same than those used in Fig. 3.

when the field changes its sign the electron has a probability to re-collide with the ion core $\mathrm{He}^{+}$. As a third step, this colliding electron can kick out the second electron if and only if the first electron kinetic energy is larger than $I_{2 p}$, the ionization potential of the second remaining electron in the ion core: RIDI or (e, 2e) mechanism. For the conventional field cases at low laser peak intensities (about $0.8-3 \times 10^{14} \mathrm{~W} / \mathrm{cm}^{2}$ ), the probability that the first electron reaches a larger enough energy as to overcome the ionization potential of the second electron is negligible. Thus, it is rather unlikely that the double ionization process be mediated by the so-called RIDI mechanism (see Sec. 1 for more details). However, from the behaviour of electrons driven by spatially inhomogeneous fields (see e.g. [15]), it is very likely that the first-ionized electron gains a much larger energy compared to the conventional case. Thus, at the instant of re-collision, the second electron would have a higher chance to be ionized in a spatially inhomogeneous field, which corresponds to an enhancement of the double electron ionization probability.

Note that according to Refs. $[10,11]$ the RIDI process is limited by the energy that the first electron can accumulate from the laser field. The calculations depicted in Figs. 3 and 4 provide enough evidence to confirm that the spatial inhomogeneous field could open this channel at a much lower laser peak intensity. This happens because the first electron gains much more energy in the field, increasing then the probability to ionize the second electron.

\subsection{Correlated two-electron momentum maps}

Another interesting observable, which contains information about the e-e correlation, is the 2e-momentum distribution. This observable has allowed to disentangle the common sequential and non-sequential double RESI, 

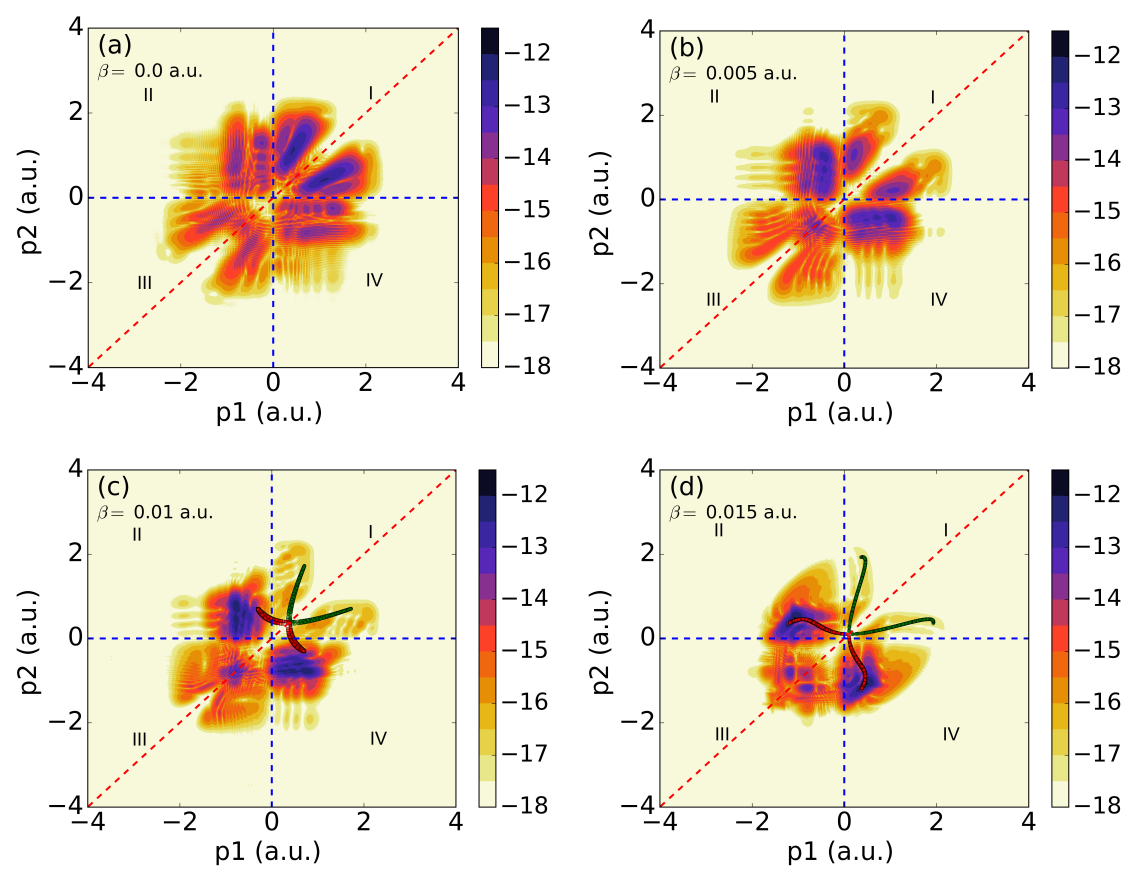

Fig. 5 Numerical two-electron momentum distribution for various inhomogeneity degrees: $\beta=0.000$ (a), 0.005 (b), 0.010 (c) and 0.015 a.u. (d). The color scale is $\log _{10}\left[S_{2 \mathrm{e}}\left(p_{1}, p_{2}\right)\right]$. Vertical and horizontal blue dashed lines denote the 2e momentum axes which help us to distinguish between correlated, (i) and (iii) quadrants, and anticorrelated, (ii) and (iv) quadrants, regions. The diagonal red dashed line $p_{1}=p_{2}$ represents the max e-e correlation momentum points or the total 2e momentum $p=p_{1}+p_{2}$. CTMC for the (e, 2e) mechanisms are superimposed in green-circles (recoil process) and in red-squares (binary process) in (c) and (d) panels. The laser peak intensity used for these numerical calculations is $I_{0}=2 \times 10^{14} \mathrm{~W} / \mathrm{cm}^{2}$. The other laser parameters are the same as those used in Fig. 3.

rescattering impact ionization and laser-field assisted rescattering ionization mechanisms $[12,13,38]$. Fig. 5 depicts $S_{2 \mathrm{e}}\left(p_{1}, p_{2}\right)$ for different $\beta$ parameters at the same fixed laser peak intensity of $I_{0}=2 \times 10^{14} \mathrm{~W} / \mathrm{cm}^{2}$. The doubleelectron map in Fig. 5(a) exhibits two large probability lobe peaks on the first quadrant of the correlation region - in almost perfect concordance with the results published in Ref. [19]. This probability distribution indicates that both electrons prefer to leave on the same (positive) direction. It is understood that the repulsive e-e Coulomb potential plays an important role at those relative low peak intensity for the $\mathrm{He}$ model [19].

Note that a classical rescattering electron scenario $(e, 2 e)$ is not good enough for describing this NSDI mechanism of our He model at this peak intensity. From a classical viewpoint, the rescattering energy $E_{\mathrm{k} \text {,max }}=$ 
$3.17 U_{p}=1.4$ a.u., is lower than the second ionization potential $I_{2 p} \sim 1.5$ a.u. This is the main reason to not compute the double electron ionization maps by means of CTMC simulations. This is so because the classical rescattering energy of the first electron is not enough to exceed the ionization potential of the second electron. Instead, these double-electron ionization maps, Figs. 5(a) and 5(b), could be understood as a laser-field-assisted rescattering process for which such a constraint does not apply $[11-13,19,38]$. As pointed out in $[9,19]$, the driving laser field provides the rest of the required energy to remove the second electron at the instant of recollision.

For further interpretations of Fig. 5 we recall that finding double ionization in quadrants I and III corresponds to both electron momenta pointing in the same direction. In contrast, quadrants II and IV contain the cases of the electrons' momenta pointing into opposite directions. When both electrons leave the atom in the same direction, we say they are correlated. Comparing the 2e-momentum distributions in Figs. 5(a) and (b), we find that the two electrons prefer to detach in opposite directions when driven by plasmonic-enhanced spatially inhomogeneous fields. This effect is even larger for an inhomogeneity degree of $\beta=0.01$ and 0.015 a.u., as can be seen in Figs. 5(c)-(d). We note, however, the appearance of a small 2e-probability also in the correlated regions.

Naturally, questions about the physical mechanisms behind those effects in the 2e maps emerge. In order to address those questions, we superimposed our CTMC calculations on the TDSE results in Figs. 5(c) and (d) for the cases of binary (red-squares) and recoil (green-circles) processes. As is observed, a reasonable agreement between the TDSE and the CTMC calculations is found. In particular, the concordance is remarkable for the case of $\beta=0.015$ a.u. This clearly corroborates that the forward rescattering process with respect to the first incident electron direction, binary, is highly probable within that so-called (e, 2e) mechanisms if spatially inhomogeneous fields drive the two-electron system. Note that this agreement of TDSE and CTMC supports our previous observation that the 2e-particles are likely to prefer leaving the atom in opposed directions.

Furthermore, according to Weber et al. [8] the momentum distribution corresponding to the coordinates $p=p_{1}+p_{2}$ (diagonal along $p_{1}=p_{2}$ ) and $p^{-}=p_{1}-p_{2}$ (diagonal $\left.p_{1}=-p_{2}\right)$, are helpful for describing the importance of two effects: e-e repulsion and acceleration of the particles by the optical field. On the one hand, e-e repulsion does not change $p$ but contributes to $p^{-}$. On the other hand, the momentum transfer received from the field is identical. So, this part of the acceleration does not change $p^{-}$but contributes to $p$. Note, however, that this statement is only valid if the electric field does not depend on the position. Thereby, for the inhomogeneous field cases we cannot conclude that the acceleration part does not contribute to $p^{-}$as it is the case in conventional fields. 

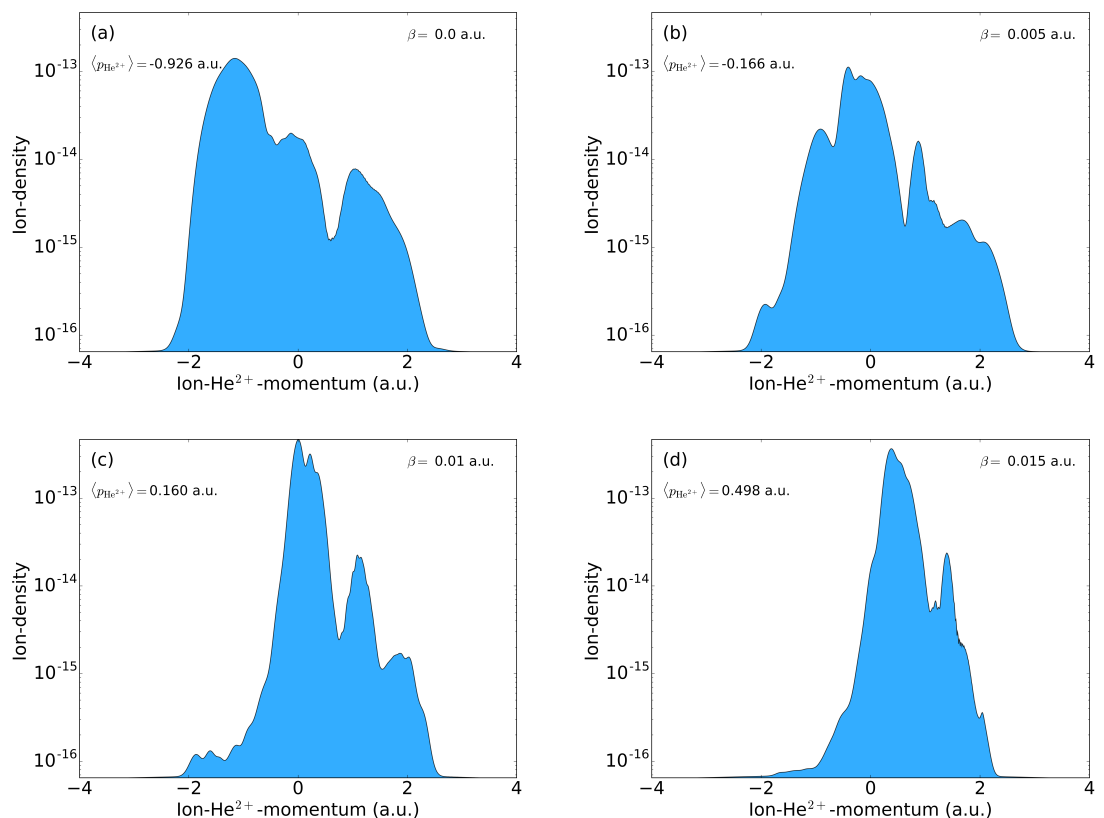

Fig. 6 Correlated ion $\mathrm{He}^{2+}$ momentum distributions, $S_{\mathrm{He}^{2+}}\left(p_{\text {ion }}\right)$, corresponding to the panels (a)-(d) of Fig. 5. We have also computed the ion expectation values $\left\langle p_{\mathrm{He}^{+2}}\right\rangle$ as a function of $\beta$ and show it in each plot (see the top left of each panel). The difference is remarkable: from negative values to positive ones. This clearly points out that the spatial inhomogeneous field configures an instrumental tool to control the ion direction emission.

This is in absolute concordance with what we observe in the $2 \mathrm{e}$ momentum maps for $\beta=0.010$ and 0.015 a.u.

Additionally, in Fig. 6 we show the correlated ion $\mathrm{He}^{2+}$ momentum distributions corresponding to the $S_{2 e}\left(p_{1}, p_{2}\right)$ panels of Fig. 5. A first observation is that a large momentum-shift is found for the recoiling ion as the inhomogeneity degree $\beta$ increases. For the conventional field case depicted in Fig. 6(a), the full momentum width of the distribution is about $\pm 2 A_{0}= \pm 2.6$ a.u., where $A_{0}=E_{0} / \omega_{0}\left(A_{0}=1.3\right.$ a.u. $)$, is the maximum peak vector potential strength $[7,19,39]$. An asymmetry in the amplitude of the ion distribution $S_{\mathrm{He}^{2+}}\left(p_{\text {ion }}\right)$ is observed at $\beta=0$ a.u. This is due to the employed laser-field being within the few-cycle regime, $\mathrm{N}=4$, see e.g. Ref. [3] about the CEP effects. However, three peaks at about $p_{\text {ion }}^{(\max )}=\left\{-A_{0}, 0,+A_{0}\right\}$ are found. These might suggest that the laser-field assisted rescattering double ionization mechanism and the RESI mechanism take place simultaneously in such a complex correlated momentum map.

In the case of inhomogeneous fields, the ion distribution shape strongly depends on the parameter $\beta$. While the inhomogene- 
ity increases, the expectation value of the ion momentum $\left\langle p_{\mathrm{He}^{2+}}\right\rangle$ is shifted from negative to positive momentum values, i.e. the momentum expectation value changes from $\left\langle p_{\mathrm{He}^{2+}}\right\rangle=-0.92$ to +0.49 a.u. (see the top left of each panel). This indicates that the ion recoils moves in a completely opposite direction compared to the conventional field case. This strong modification in the ion direction emission, in principle experimentally detectable, is a signature of the spatial inhomogeneous character of the driven field in the DI process. In addition, the several peaks that appear in the ion distributions, suggest the possibility of different interference paths in the DI process driven by the spatially inhomogeneous field.

So far, we have studied double ionization in He via scanning the 2emomentum distribution over the inhomogeneity parameter $\beta$ at a fixed laser intensity. In order to obtain an insight about the $2 \mathrm{e}$ ionization when the laser-peak intensity increases, we compute and compare the momentummomentum distributions for the conventional $\beta=0$ and inhomogeneous $\beta=0.005$ a.u. fields. Additionally, our $a b$-initio TDSE calculations are compared with the CTMC simulations. The results are depicted in Fig. 7. While the peak intensity increases from 3 to $7 \times 10^{14} \mathrm{~W} / \mathrm{cm}^{2}$ for conventional fields, some pronounced lobes in the correlation regions are observed. Furthermore, large probability lobes in the anticorrelated region are also visible. This is a signal that the e-e Coulomb repulsion force is losing its importance while the laser-field peak intensity increases. In particular, that effect is larger for the highest intensity. In addition, note that a better agreement between TDSE and CTMC is found in the cases of Figs. $7(\mathrm{c})$ and (e) as it is expected $[7,11,19]$. This indicates that the (e, 2e) processes are the main mechanisms behind those calculations. However, in Fig. 7(a), a laser-field assisted rescattering DI process still dominates over the RIDI mechanisms. This is concluded from the poor agreement between the quantum mechanical and the classical calculations for the binary and recoil processes.

On the other hand, for inhomogeneous fields, Fig. 7(b), (d) and (f), the probability of $2 \mathrm{e}$ ionization with opposite momenta increases. This clearly indicates that the propagation of electrons under the influence of plasmonic field changes completely the 2e-dynamics. Note, that a signal in quadrant III of the correlation region is also observed, which is an indication that both electrons, independently of the incident direction of the first colliding particle, prefer to leave with negative momenta directions. Furthermore, while the laser peak intensity increases, the V-like shape in the quadrant III tends to be much closed, and also a strong signal along the diagonal $p_{1}=p_{2}$ for $p_{1}<0$ is clearly observed. These facts are the signature of e-e correlation effects rapidly losing importance while the particles are propagating in the plasmonic field. Note, however, that e-e repulsion somehow is still present because of the large momentum density width along the diagonal $p_{1}=-p_{2}$. 

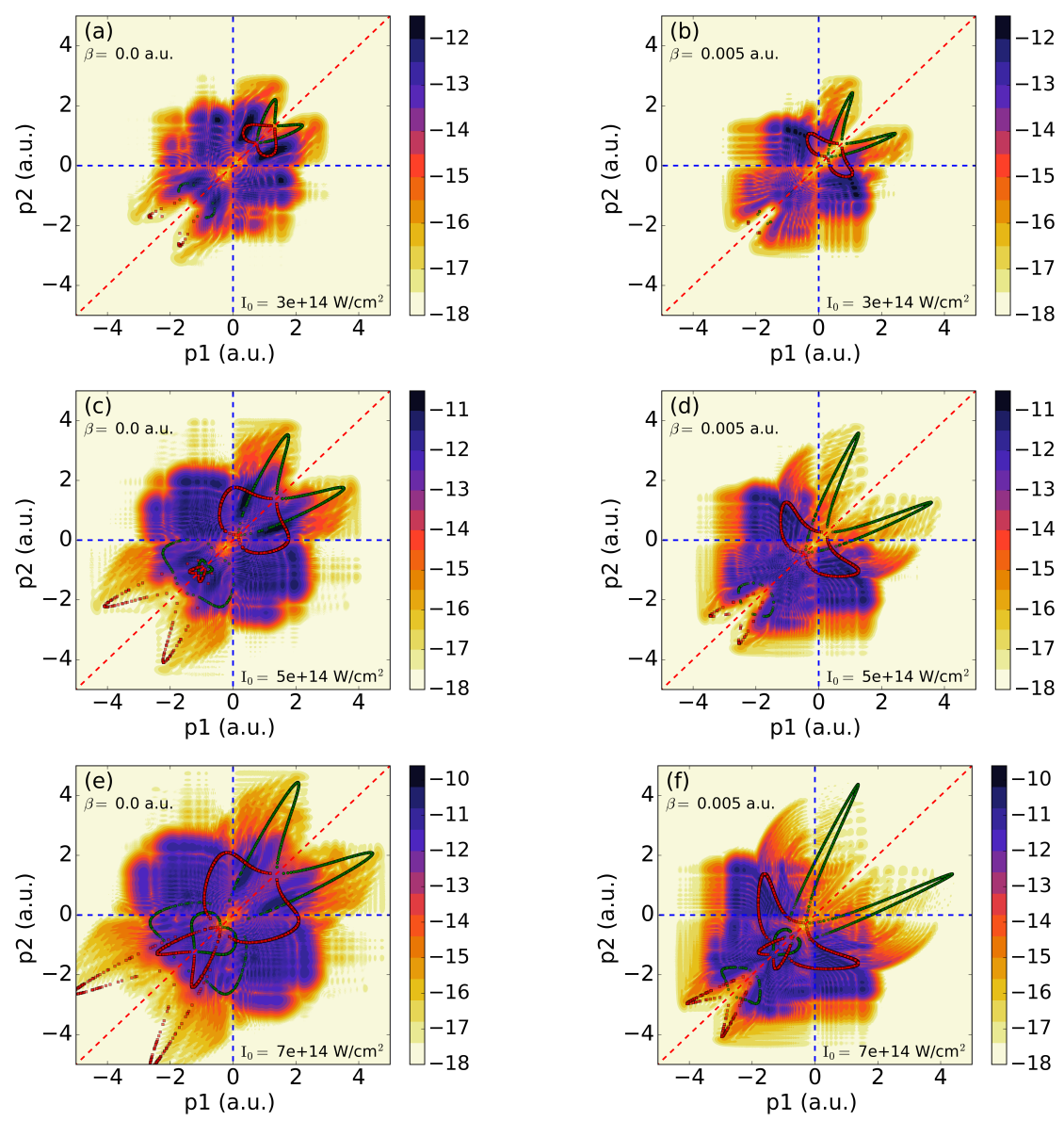

Fig. 7 Numerical two-electron momentum distributions driven by homogeneous $\beta=0$ (a), (c), (e), and inhomogeneous fields (b), (d), (e) with $\beta=0.005$ a.u., for three different laser-peak intensities: $I_{0}=3,5,7 \times 10^{14} \mathrm{~W} / \mathrm{cm}^{2}$. The CTMC calculations for binary (red-squares) and recoil (green-circles) processes are superimposed on the 2e momentum maps. Other laser parameters are the same as in Fig. 3.

Finally, it is interesting to point out that up to $7 \times 10^{14} \mathrm{~W} / \mathrm{cm}^{2}$ the NSDI by inhomogeneous fields is still within the rescattering (e, 2e) scenario. This statement is supported by the CTMC simulations that agree very well with the TDSE calculations for all studied cases. This demonstrates that the isolation of binary and recoil processes is very sensitive to the laser peak intensity. We should note, however, that between 2 and $5 \times 10^{14} \mathrm{~W} / \mathrm{cm}^{2}$, we ensure that those backward and forward rescattering processes could be separated, just by observing the anticorrelating and correlating regions of the momentum-momentum distribution. 


\section{Conclusions}

Non-sequential double ionization of helium atoms driven by a plasmonicenhanced spatially inhomogeneous fields has been theoretically investigated. By means of the fully numerical solution of the time-dependent Schrödinger equation, we observed that ion yield of $\mathrm{He}^{2+}$ substantially increases while the inhomogeneous field drives the system. An analysis of the single- and double-electron time-evolution probabilities and the two-electron momentum distribution simulations of the binary an recoil mechanisms support that the main reason for this enhancement corresponds to a high accumulated energy of the first re-colliding electron when it is moving in the spatially inhomogeneous field.

An unexpected $(\mathrm{e}, 2 \mathrm{e})$ mechanism at very low intensity, i.e. $I_{0}=2 \times$ $10^{14} \mathrm{~W} / \mathrm{cm}^{2}$ is observed with increasing the inhomogeneity strength. Note that the double electron ionization effects induced by the plasmonicenhanced fields will depend on (i) the peak intensity; (ii) the spatial properties of the field and (iii) the applied target. The latter is so because of the different ionization potentials for different atomic and molecular species. This means that both by engineering the inhomogeneous field and controlling the laser intensity the two different mechanisms, namely the laser-field-assisted re-scattering and the RIDI process can be isolated. Furthermore, our interpretation of the fully abinitio TDSE for the two-electron momentum distributions by comparing to CTMC simulations, allowed us to distinguish between binary and recoil processes if and only if the spatially inhomogeneous field drives the system.

Furthermore, the spatial characteristics of the plasmonic-enhanced field break the symmetry of the 2e acceleration in the anticorrelated region. This is noted in the pronounced peaked probability of the two-electron momentum distribution located in the II and IV quadrants. Physically this is translated by the fact that the two-electron propagation is much more affected by the plasmonic field than by the e-e correlation at the double-ionization time. Thereby, plasmonic-enhanced fields configure an interesting alternative to control correlation effects in the double ionization process.

Still there are open questions, e.g. concerning the role of the e-e Coulomb potential while both identical particles are propagating within the spatially inhomogeneous field and how this effect is related to the 2e momentum distribution maps for larger laser peak intensities here used. We plan to address these questions in subsequent work.

\section{Acknowledgements}

This work was supported by the project ELI- Extreme Light Infrastructurephase 2 (Project No. CZ.02.1.01/0.0/0.0/15_008/0000162) from European 
Regional Development Fund, Ministerio de Economía y Competitividad through Plan Nacional [Grant No. FIS2011-30465-C02-01, FrOntiers of QUantum Sciences (FOQUS): Atoms, Molecules, Photons and Quantum Information Grants No. FIS2013-46768-P and No. FIS2014-56774-R; and Severo Ochoa Excellence Grant No. SEV-2015-0522], the Catalan Agencia de Gestio d'Ajuts Universitaris i de Recerca (AGAUR) with SGR 874 2014-2016, Fundació Privada Cellex Barcelona. N.S. was supported by the Erasmus Mundus Doctorate Program Europhotonics (Grant No. 159224-1-2009-1FR-ERA MUNDUS-EMJD). N.S., A.C., and M.L. acknowledge ERC AdG OSYRIS, EU FETPRO QUIC and National Science Centre, Poland - Symfonia grant 2016/20/W/ST4/00314. A. S. L. and L. O. acknowledge Max Planck Center for Attosecond Science (MPC-AS). J. A. P.-H. acknowledges to the Spanish Ministerio de Economía y Competitividad (FURIAM Project No. FIS2013-47741-R and PALMA project FIS2016- 81056-R) and LaserlabEurope (EU-H2020 654148).

\section{References}

1. A. L'Huillier, L. A. Lompre, G. Mainfray, and C. Manus. Multiply charged ious formed by multiphoton absorption processes in the continuum. Phys. Rev. Lett., 48(26):1814-1817, (1982).

2. P. Lambropoulos, X. Tang, P. Agostini, G. Petite, and A. L'Huillier. Multiphoton spectroscopy of doubly excited, bound, and autoionizing states of strontium. Phys. Rev. A, 38(12):6165-6179, (1988).

3. B. Bergues, M. Kübel, N. G. Kling, C. Burger, and M. F. Kling. Singlecycle non-sequential double ionization. IEEE J. Sel. Top. Quantum Electron., 21(5):8701009-8701009, (2015).

4. B. Bergues, M. Kübel, N. G. Johnson, B. Fischer, N. Camus, K. J. Betsch, O. Herrwerth, A. Senftleben, A. M Sayler, T. Rathje, T. Pfeifer, I. Ben-Itzhak, R. R. Jones, G. G. Paulus, F. Krausz, R. Moshammer, J. Ullrich, and M. F. Kling. Attosecond tracing of correlated electron-emission in non-sequential double ionization. Nature Comm., 3(813):1-6, (2012).

5. M. G. Pullen, B. Wolter, X. Wang, X.-M. Tong, M. Sclafani, M. Baudisch, H. Pires, C. D. Schröter, J. Ullrich, T. Pfeifer, R. Moshammer, J. H. Eberly, and J. Biegert. Transition from non-sequential to sequential double ionisation in many-electron systems. ArXiv:1602.07840, (2016).

6. B. Walker, B. Sheehy, L. F. DiMauro, P. Agostini, K. J. Schafer, and K. C. Kulander. Precision measurement of strong field double ionization of helium. Phys. Rev. Lett., 73(9):1227-1230, (1994).

7. Th. Weber, M. Weckenbrock, A. Staudte, L. Spielberger, O. Jagutzki, V. Mergel, F. Afaneh, G. Urbasch, M. Vollmer, H. Giessen, and R. Dörner. Recoil-ion momentum distributions for single and double ionization of helium in strong laser fields. Phys. Rev. Lett., 84(3):443-446, (2000).

8. Th. Weber, H. Giessen, M. Weckenbrock, G. Urbasch, A. Staudte, L. Spielberger, O. Jagutzki, V. Mergel, M. Vollmer, and R. Dörner. Correlated electron emission in multiphoton double ionization. Nature, 405(6787):658-661, (2000). 
9. W. Becker, X.J. Liu, P. J. Ho, and J. H. Eberly. Theories of photoelectron correlation in laser-driven multiple atomic ionization. Rev. Mod. Phys., 84(3):1011-1043, (2012).

10. P. B. Corkum. Plasma perspective on strong-field multiphoton ionization. Phys. Rev. Lett., 71(13):1994-1997, (1993).

11. A. Staudte, C. Ruiz, M. Schöffler, S. Schössler, D. Zeidler, Th. Weber, M. Meckel, D. M. Villeneuve, P. B. Corkum, A. Becker, and R. Dörner. Binary and recoil collisions in strong field double ionization of helium. Phys. Rev. Lett., 99(26):263002, (2007).

12. R. Kopold, W. Becker, H. Rottke, and W. Sandner. Routes to nonsequential double ionization. Phys. Rev. Lett., 85(18):3781-3784, (2000).

13. E. Eremina, X. Liu, H. Rottke, W. Sandner, A. Dreischuh, F. Lindner, F. Grasbon, G. G. Paulus, H. Walther, R. Moshammer, B. Feuerstein, and J. Ullrich. Laser-induced non-sequential double ionization investigated at and below the threshold for electron impact ionization. J. Phys. B: At. Mol. Phys, 36(15):3269-3280, (2003).

14. A. Chacón, M. F. Ciappina, and M. Lewenstein. Double-electron recombination in high-order-harmonic generation driven by spatially inhomogeneous fields. Phys. Rev. A, 94(4):043407, (2016).

15. M. F. Ciappina, J. A. Pérez-Hernández, A. S. Landsman, W. Okell, S. Zherebtsov, B. Förg, J. Schötz, L. Seiffert, T. Fennel, T. Shaaran, T. Zimmermann, R. Guichard A. Chacón, A. Zaïr, J. W. G. Tisch, J. P. Marangos, T. Witting, A. Braun, S. A. Maier, L. Roso, M. Krüger, P. Hommelhoff, M. F. Kling, F. Krausz, and M. Lewenstein. Attosecond physics at the nanoscale, (in press). Arxiv1607.01480.

16. D. Bauer. Two-dimensional, two-electron model atom in a laser pulse: Exact treatment, single-active-electron analysis, time-dependent density-functional theory, classical calculations, and nonsequential ionization. Phys. Rev. A, 56(4):3028-3039, (1997).

17. D. G. Lappas and R. van Leeuwen. Electron correlation effects in the double ionization of he. J. Phys. B, 31(6):L249-L256, (1998).

18. D. G. Lappas, A. Sanpera, J. B. Watson, K. Burnett, P. L. Knight, R. Grobe, and J. H. Eberly. Two-electron effects in harmonic generation and ionization from a model he atom. J. Phys. B: At. Mol. Phys, 29(16):L619, (1996).

19. M. Lein, E. K. U. Gross, and V. Engel. Intense-field double ionization of helium: Identifying the mechanism. Phys. Rev. Lett., 85(22):4707-4710, (2000).

20. S. E. Harris, J. J. Macklin, and T. W. Hänsch. Atomic scale temporal structure inherent to high-order harmonic generation. Opt. Comm., 100(5-6):487-490, (1993).

21. C.G. Wahlström, M.B. Gaarde, A. L'Huillier, C. Lyngå, I. Mercer, E. Mevel, R. Zerne, Ph. Antoine, M. Bellini, and T.W. Hänsch. Manipulations of highorder harmonics. In P. Lambropoulos and H. Walther, editors, Multiphoton Processes 1996, page 160. Institute of Physics Publishing, Bristol, 1997.

22. R. Zerne, C. Altucci, M. Bellini, M. B. Gaarde, T. W. Hänsch, A. L'Huillier, C. Lyngå, and C.-G. Wahlström. Phase-locked high-order harmonic sources. Phys. Rev. Lett., 79(6):1006-1009, (1997).

23. J. B. Watson, A. Sanpera, D. G. Lappas, P. L. Knight, and K. Burnett. Nonsequential double ionization of helium. Phys. Rev. Lett., 78(10):18841887, (1997). 
24. A. Chacón, M. F. Ciappina, and M. Lewenstein. Numerical studies of lightmatter interaction driven by plasmonic fields: The velocity gauge. Phys. Rev. $A, \mathbf{9 2}(6): 063834,(2015)$.

25. M. F. Ciappina, J. Biegert, R. Quidant, and M. Lewenstein. High-orderharmonic generation from inhomogeneous fields. Phys. Rev. A, 85(3):033828, (2012).

26. M. D. Feit, J. A. Fleck, and A. Steiger. Solution of the schrdinger equation by a spectral method. J. Comput. Phys., 47(3):412-433, (1982).

27. C. Ruiz and A. Chacón. QFISHBOWL library, (2008). http://code.google.com/p/qfishbowl.

28. M. Frigo and S. G. Johnson. FFTW library, (1998). http://www.fftw.org.

29. R. Grobe and J. H. Eberly. One-dimensional model of a negative ion and its interaction with laser fields. Phys. Rev. A, 48(6):4664-4681, (1993).

30. M. V. Ammosov, N. B. Delone, and V. P. Krainov. Tunnel ionization of complex atoms and of atomic ions in an alternating electromagnetic field. Sov. Phys.-JETP, 64(6):1191-1194, (1986).

31. N. B. Delone and V. P. Krainov. Energy and angular electron spectra for the tunnel ionization of atoms by strong low-frequency radiation. J. Opt. Soc. Am. B, 8(6):1207-1211, (1991).

32. L. Arissian, C. Smeenk, F. Turner, C. Trallero, A. V. Sokolov, D. M. Villeneuve, A. Staudte, and P. B. Corkum. Direct test of laser tunneling with electron momentum imaging. Phys. Rev. Lett., 105(13):133002, (2010).

33. A. S. Landsman, A.N. Pfeiffer, C. Hofmann, M. Smolarski, C. Cirelli, and U. Keller. Rydberg state creation by tunnel ionization. New J. Phys., 15(1):013001, (2013).

34. A. S. Landsman and U. Keller. Tunnelling time in strong field ionisation. J. Phys. B: At. Mol. Phys, 47(20):204024, (2014).

35. T. Nubbemeyer, K. Gorling, A. Saenz, U. Eichmann, and W. Sandner. Strongfield tunneling without ionization. Phys. Rev. Lett., 101(23):233001, (2008).

36. C. Hofmann, A. S. Landsman, C. Cirelli, A. N. Pfeiffer, and U. Keller. Comparison of different approaches to the longitudinal momentum spread after tunnel ionization. J. Phys. B: At. Mol. Phys, 46(12):125601, (2013).

37. S. P. Goreslavski, G. G. Paulus, S. V. Popruzhenko, and N. I. ShvetsovShilovski. Coulomb asymmetry in above-threshold ionization. Phys. Rev. Lett., 93(2):233002, (2004).

38. Y. Liu, S. Tschuch, A. Rudenko, M. Dürr, M. Siegel, U. Morgner, R. Moshammer, and J. Ullrich. Strong-field double ionization of ar below the recollision threshold. Phys. Rev. Lett., 101(5):053001, (2008).

39. A. Rudenko, K. Zrost, B. Feuerstein, V. L. B. de Jesus, C. D. Schröter, R. Moshammer, and J. Ullrich. Correlated multielectron dynamics in ultrafast laser pulse interactions with atoms. Phys. Rev. Lett., 93(25):253001, (2004). 